\title{
Vivid: How valence and arousal influence word processing under different task demands
}

\author{
Nathaniel Delaney-Busch ${ }^{1}$ - Gianna Wilkie ${ }^{1,2}$ • Gina Kuperberg ${ }^{1,3}$
}

Published online: 29 January 2016

(C) Psychonomic Society, Inc. 2016

\begin{abstract}
In this study, we used event-related potentials to examine how different dimensions of emotion-valence and arousal - influence different stages of word processing under different task demands. In two experiments, two groups of participants viewed the same single emotional and neutral words while carrying out different tasks. In both experiments, valence (pleasant, unpleasant, and neutral) was fully crossed with arousal (high and low). We found that the task made a substantial contribution to how valence and arousal modulated the late positive complex (LPC), which is thought to reflect sustained evaluative processing (particularly of emotional stimuli). When participants performed a semantic categorization task in which emotion was not directly relevant to task performance, the LPC showed a larger amplitude for high-arousal than for low-arousal words, but no effect of valence. In contrast, when participants performed an overt valence categorization task, the LPC showed a large effect of valence (with unpleasant words eliciting the largest positivity), but no effect of arousal. These data show not only that valence and arousal act independently to influence word processing, but that their
\end{abstract}

Electronic supplementary material The online version of this article (doi:10.3758/s13415-016-0402-y) contains supplementary material, which is available to authorized users.

Nathaniel Delaney-Busch

Nathaniel.Delaney-Busch@tufts.edu

1 Department of Psychology, Tufts University, 490 Boston Ave, Medford, MA 02155, USA

2 University of Massachusetts Medical School, Worcester, MA, USA

3 Department of Psychiatry and Athinoula A. Martinos Center for Biomedical Imaging, Massachusetts General Hospital, Harvard Medical School, Boston, MA, USA relative contributions to prolonged evaluative neural processes are strongly influenced by the situational demands (and by individual differences, as revealed in a subsequent analysis of subjective judgments).

Keywords Event-related potentials · ERP - LPC · LPP - Late positivity $\cdot$ Emotion $\cdot$ Valence $\cdot$ Arousal $\cdot$ Language $\cdot$ Word processing $\cdot$ Task

Emotional stimuli elicit a rapid and coordinated set of responses. They can capture attention, guide evaluative judgments, and mobilize autonomic reflexes quickly and powerfully. These emotion responses are generally described as having an appetitive/aversive direction, called valence, and a level of activation, called arousal. In this study, we used event-related potentials (ERPs), a direct measure of online neural activity, to ask how valence and arousal influence different stages of emotional word processing under different tasks demands.

\section{General Introduction}

According to the circumplex model of emotion, valence and arousal reflect two orthogonal underlying dimensions of emotion, which together explain most of the variation in how emotional stimuli are evaluated (Abelson \& Sermat, 1962; Osgood, Suci, \& Tannenbaum, 1967; Russell, 1980). The valence dimension ranges from pleasant to neutral to unpleasant, and reflects the general motivational significance of a stimulus. ${ }^{1}$ The arousal

\footnotetext{
${ }^{1}$ In the present article, we will use pleasant and unpleasant to express the levels of valence, in order to distinguish them from positive and negative ERP voltages. This terminology is also recommended in Watson and Tellegen's (1985) proposed naming conventions for a bipolar valence dimension.
} 
dimension ranges from high (or active) to low (or passive), and expresses the degree to which a particular stimulus prepares a person for action - for example, by eliciting an autonomic sympathetic response (Bradley \& Lang, 2007) or capturing and focusing attention (Mather \& Sutherland, 2011).

Although there is evidence that the dimensions of valence and arousal can explain unique variance across explicit evaluative ratings of mood, facial expressions, and words (Russell, 1980), in practice these ratings are consistently correlated with one another: Stimuli that are overtly rated as extremely valenced (pleasant or unpleasant) also tend to be rated as highly arousing, whereas stimuli rated as less valenced (more neutral) tend to be rated as less arousing, leading to a "U-shaped" relationship between the two (Lang, Bradley, \& Cuthbert, 2005). This close correlation between overt evaluations of valence and arousal raises a straightforward question: Does the brain actually distinguish between these two dimensions of emotion during word processing? Or, alternatively, does arousal fall naturally out of the dimension of valence, such that as "pleasantness" or "unpleasantness" increases, so does arousal?

To address this question, several researchers have investigated whether arousal and valence exert independent effects on behavior. There is now fairly strong evidence that, even when valence is held constant (Aquino \& Arnell, 2007; Arnell, Killman, \& Fijavz, 2007), high-arousal stimuli capture and hold attention more than low-arousal stimuli (Anderson, 2005; Mather \& Sutherland, 2011; Vogt, De Houwer, Koster, Van Damme, \& Crombez, 2008), in addition to eliciting systemic arousal (Bradley \& Lang, 2007). The effects of valence, independent of arousal, however, have been more mixed. Some studies have reported larger or stronger attentional and autonomic effects of unpleasant than of pleasant stimuli that are matched on arousal (Peeters \& Czapinski, 1990). This is known as a negativity bias (Baumeister, Bratslavsky, Finkenauer, \& Vohs, 2001; Ito, Larsen, Smith, \& Cacioppo, 1998; Taylor, 1991): the "tendency for the negative motivational system to respond more intensely than the positive motivational system to comparable increases in input" (Ito \& Cacioppo, 2005, p. 2). Others, however, have reported no difference between the behavioral responses to pleasant and unpleasant stimuli, or even stronger responses to pleasant stimuli (see Kousta, Vinson, \& Vigliocco, 2009, for a discussion of these inconsistencies).

\section{ERP studies}

Behavioral responses like those discussed above typically reflect the culmination of multiple stages of neural processing. An alternative approach would be to directly measure the neural activity evoked by a given stimulus as it is processed in real time. Event-related potentials (ERPs) enable just this, and a number ERP studies have now examined the neurocognitive processes recruited in response to emotional stimuli, including words.
Some of these studies have reported very early effects of emotion, within the first $150 \mathrm{~ms}$ of word onset, with effects reported on the N1 component (Briesemeister, Kuchinke, \& Jacobs, 2014; Hinojosa, Méndez-Bértolo, \& Pozo, 2010; Hofmann, Kuchinke, Tamm, Võ, \& Jacobs, 2009; Kissler \& Herbert, 2013; Wang, Bastiaansen, Yang, \& Hagoort, 2013), the P2 component (González-Villar, Triñanes, Zurrón, \& Carrillo-de-laPeña, 2014; Herbert, Kissler, Junghöfer, Peyk, \& Rockstroh, 2006; Kanske \& Kotz, 2007; Ortigue et al., 2004; Wang et al., 2013), or on other early perceptual components (Bayer, Sommer, \& Schacht, 2012; Bernat, Bunce, \& Shevrin, 2001; Keuper et al., 2014; Scott, O’Donnell, Leuthold, \& Sereno, 2009; Zhang et al., 2014). This has been taken to reflect a very early influence of emotion on initial perceptual processing. These findings, however, have been quite variable, with the majority of such studies failing to find such early effects. ${ }^{2}$

The most consistent effect of emotion in ERP studies has been on a positive-going ERP component that begins around 400-500 ms after word onset and extends for several hundred milliseconds (Citron, 2012; Hajcak, MacNamara, \& Olvet, 2010; Kissler, Assadollahi, \& Herbert, 2006)—-henceforth referred to as the emotional late positive complex (LPC). Although there is debate about the precise functional significance of the emotional LPC (Gable, Adams, \& Proudfit, 2015), for the purposes of this article, we assume that it is likely related to the sustained evaluation of the motivational significance of a salient stimulus (Hajcak et al., 2010; Hajcak, Weinberg, MacNamara, \& Foti, 2012; Weinberg \& Hajcak, 2011). As such, the emotional LPC has been theorized to be part of the P300 family of components (Crites, Cacioppo, Gardner, \& Berntson, 1995; Delplanque, Silvert, Hot, Rigoulot, \& Sequeira, 2006), which can reflect the attentional demands of evaluating task-relevant oddball or other similarly salient stimuli (Donchin \& Coles, 1988; Nieuwenhuis, AstonJones, \& Cohen, 2005; Polich, 2012). Importantly, just as for the $\mathrm{P} 300$, the sustained evaluative processing reflected by the emotional LPC is not static, but depends on both task and context (Dunning \& Hajcak, 2009; Fields \& Kuperberg, 2012; Fischler \& Bradley, 2006; Hajcak, Dunning, \& Foti, 2009; Holt, Lynn, \& Kuperberg, 2009; Schindler, Wegrzyn, Steppacher, \& Kissler, 2014; Schupp et al., 2007).

Several studies have reported a larger LPC on high- than on low-arousal words (and pictures: Leite et al., 2012), even

\footnotetext{
${ }^{2}$ Other ERP studies have reported reliable effects of emotion on an early posterior negativity (or EPN), a temporo-parietal component that emerges between 200 and $300 \mathrm{~ms}$ poststimulus (Citron, 2012). This has been interpreted as reflecting a prioritized deployment of selective attention toward emotional meaning at an early stage of lexico-semantic processing. However, this is generally seen when data are analyzed using wholebrain average reference electrodes, rather than when using mastoid references (Olofsson, Nordin, Sequeira, \& Polich, 2008). The electrode array and averaged mastoid references used in the present experiments were chosen to capture the LPC well, but this setup precluded appropriate analysis of the EPN.
} 
when valence is kept constant (Bayer et al., 2012; Delplanque et al., 2006; Recio, Conrad, Hansen, \& Jacobs, 2014), though others have revealed no differences (Bayer, Sommer, \& Schacht, 2010) or more complex interactions between valence and arousal (Citron, Weekes, \& Ferstl, 2013). Importantly, two recent studies (Bayer et al., 2012; Recio et al., 2014) suggested that the LPC arousal effect can also be elicited using neutrally valenced stimuli, in which high-arousal words like "joust," "scrimmage," and "samurai" elicit a larger LPC amplitude than low-arousal words like "table" and "sculpture." High-arousal neutral words have not traditionally been included in many behavioral or ERP studies of emotion processing. However, they are an important component of the circumplex model (Russell, 1980), in which variation in arousal is distinct from variation in valence. Though these dimensions of emotion were derived primarily from factor analyses of how moods and emotional stimuli were overtly evaluated, it is plausible that the circumplex model may also describe how emotional stimuli are actually processed, and the relative orthogonality of valence and arousal processing would be an important indicator of this effect.

There is also evidence for effects of valence on the LPC, independent of arousal. Most studies have reported a larger LPC on valenced words (both pleasant and unpleasant) than on neutral words that are matched for low levels of arousal (Citron, 2012). Others have reported a negativity bias, with a larger (Ito et al., 1998) and/or longer (Hajcak \& Olvet, 2008) LPC to unpleasant than to pleasant stimuli matched for high levels of arousal (for examples using word stimuli, see Delaney-Busch \& Kuperberg, 2013; Fields \& Kuperberg, 2012; Holt et al., 2009). Other studies, however, have described larger LPC amplitudes on pleasant than on unpleasant arousal-matched stimuli (Bayer et al., 2012; Briggs \& Martin, 2009; Herbert, Junghöfer, \& Kissler, 2008; Kissler \& Herbert, 2013; Kissler, Herbert, Winkler, \& Junghöfer, 2009; Recio et al., 2014), and still others have reported an interactive pattern between valence and arousal (Citron et al., 2013; Feng et al., 2014) or no effects at all.

In sum, the existing data suggest that although both arousal and valence can independently contribute to behavioral and neural responses, these effects are quite variable. There could be several possible reasons for this variability. For instance, some studies have not controlled for potentially confounding factors (discussed by Kissler et al., 2006), such as orthographic neighborhood or concreteness (e.g., Scott et al., 2009; Zhang et al., 2014), although others have used very wellcontrolled stimuli (e.g., Bayer et al., 2012; Recio et al., 2014). Most relevant to the questions of the present study, previous investigations have used different tasks that imposed quite different situational demands.

Furthermore, task goals have long been known to influence behavioral reactions to emotional stimuli (see Lai, Hagoort, \& Casasanto, 2012, for a particularly elegant example). And, as we noted above, consistent with its relationship with the P300, task is also known to modulate the amplitude of the LPCs evoked by emotional stimuli (Dunning \& Hajcak, 2009; Hajcak et al., 2009; Hajcak, Moser, \& Simons, 2006; Hajcak et al., 2012). For example, attending to nonemotional features of words, such as during a word/ nonword lexical-decision task, reduces the overall effect of emotion on the LPC, while attending to emotional features, such as during explicit valence judgments, increases the effect (Fischler \& Bradley, 2006).

\section{The present study}

Although previous studies provided evidence that the emotional relevance of a task can increase sustained evaluative processing to emotional stimuli, it remains unclear whether or how such sustained evaluative processing, as reflected by the $\mathrm{LPC}$, is differentially influenced by the task relevance of the dimensions of emotion: valence and arousal. For instance, how does the arousal effect change when valence is made task-relevant (vs. when neither is task-relevant)?

To address these questions, we carried out two ERP experiments using identical sets of stimuli but different tasks. In Experiment 1, participants performed a semantic-monitoring task in which neither the valence nor the arousal properties of the words were overtly task-relevant. In Experiment 2, participants explicitly judged the valence of each word. In both experiments, we used a $3 \times 2$ factorial design that fully crossed three levels of valence (pleasant, unpleasant, and neutral) with two levels of arousal (high and low). This design meant that we not only included low-arousal, but also high-arousal neutral words. Such high-arousal neutral words consistently appear in large rating studies (Bradley \& Lang, 1999; Redondo, Fraga, Padrón, \& Comesaña, 2007; Võ, Jacobs, \& Conrad, 2006; Warriner, Kuperman, \& Brysbaert, 2013). Although debate remains concerning their precise functional implications (see the General Discussion), these high arousal ratings cannot be easily explained by simple valence ambiguity (Bayer, Sommer, \& Schacht, 2011) and can be empirically identified in the same manner as any other condition. ${ }^{3}$ Finally, we also controlled for a number of possibly confounding features across our six conditions, including frequency, concreteness, word length, orthographic neighborhood size, bigram frequency, and word class.

Our primary focus for these experiments was on the specific contributions of valence and arousal to word processing in the LPC, which reflects sustained evaluative processing. We considered two broad possibilities for the influence of task relevance. The first was that similar effects of valence and

\footnotetext{
${ }^{3}$ We do not intend to argue that arousal is a meaningful concept in the absence of motivational significance (see the General Discussion).
} 
arousal would be seen in both tasks. This would indicate that sustained evaluative processing of each emotion dimension would be evoked by the inherent emotional properties of words, regardless of their task relevance (e.g., as a necessary consequence of emotion perception). The second overall possibility was that the relative effects of valence and arousal on the LPC would differ depending on task demands. For example, in Experiment 1, in which emotion was irrelevant to task performance, any sustained evaluative processing might be driven more by the arousal properties of the words than by their valence (as we discuss below). In Experiment 2, however, in which valence was overtly task-relevant, sustained evaluative processing might be driven more by valence (González-Villar et al., 2014) than by arousal. These findings would indicate that the sustained evaluative processing evoked by emotional stimuli is dynamic, reflecting in part the relevance of the valence and arousal dimensions to the current situational demands.

\section{Experiment 1}

In Experiment 1 we aimed to determine when and how the valence and arousal properties of words would influence neural processing when they were both irrelevant to task performance. A large body of literature now suggests that emotional stimuli can capture attention and distract from the task requirements in many different experimental contexts (Carretié, 2014; Lang, Bradley, \& Cuthbert, 1997; Okon-Singer, Lichtenstein-Vidne, \& Cohen, 2013). This is the case even when participants are explicitly asked to ignore the emotional features of such stimuli (Arnell et al., 2007). Much of this "distracting" effect has been attributed to the arousal properties of emotional stimuli (Mather \& Sutherland, 2011). In support of this idea, previous ERP studies using a lexicaldecision task reported an effect of arousal (high $>$ low) on the LPCs evoked by both valenced (pleasant and unpleasant) words (Carretié et al., 2008; Hinojosa et al., 2010; Hofmann et al., 2009; Kanske \& Kotz, 2007) and neutral words (Bayer et al., 2012; Recio et al., 2014). Clearly, arousal does not need to be task-relevant in order to elicit evaluative processing.

What is less clear, however, is whether the valence properties of words can also elicit sustained evaluative processing when they are not relevant to task performance, and whether such processing is also dependent on arousal (e.g., whether both high- and low-arousal words would show the same valence effects when valence was not task-relevant). Some previous ERP studies using lexical-decision tasks have reported no effect of valence on the LPCs evoked by words that are matched on arousal (Carretié et al., 2008; Hinojosa et al., 2010; Hofmann et al., 2009; Kanske \& Kotz, 2007). Others, however, have reported a larger LPC on pleasant than on unpleasant arousal-matched words (Bayer et al., 2012; Recio et al., 2014) - a "positivity bias" - with no clear interaction between arousal and valence.

Most of these previous studies have used a lexical-decision task. Categorizing letter strings as words or nonwords, however, does not require full semantic processing (Milberg \& Blumstein, 1981): It is possible to decide whether a string of letters is a word or a nonword through recognition of familiar orthographic or phonological features. Moreover, there is evidence that valence information can actually help participants with lexical categorization (Kissler \& Herbert, 2013).

To encourage deep semantic processing, in our first experiment, we used a semantic-monitoring task in which participants had to decide whether or not each word referred to an animal, and to press a button whenever they saw such a word (animal words were not analyzed, but were added as fillers; see, e.g., Kreher, Goff, \& Kuperberg, 2009). This task therefore required participants to access the semantic features of all words and categorize all words according to group membership. However, neither valence nor arousal was directly relevant to task performance (such a task was also utilized by Fischler \& Bradley, 2006).

On the basis of the previous literature described above, we predicted that high-arousal words would capture attention and elicit sustained evaluative processing, manifesting as a larger LPC to high-arousal than to low-arousal words, regardless of valence. The key question we asked was whether valence would also capture attention and trigger sustained evaluative processing under these task conditions. We considered three possibilities. The first was that valence would act independently of arousal to influence the LPC (e.g., a positivity bias or negativity bias effect, as with a lexical-decision task; see Bayer et al., 2012; Recio et al., 2014). The second was that valence would enhance any effect of arousal, leading to a larger arousal LPC effect on valenced words than on neutral words, indicating that only valenced words might elicit strong arousal effects under such conditions. The third was that valence would have no effect at all on the LPC, indicating that when emotion is simply a distraction for the task at hand, arousal effects prevail and valence effects are minimized during the LPC time window.

\section{Method}

\section{Construction of stimuli}

Valence (pleasant, unpleasant, neutral) was fully crossed with arousal (high, low) to generate six conditions in total; see the bottom of Table 1 for examples. To generate words for each category of valence and arousal, a series of rating studies were carried out with participants who did not take part in the ERP experiment. We also collected ratings of concreteness in order to match our six experimental conditions (see below). Some ratings had been collected in a similar manner for previous published experiments (Delaney-Busch \& Kuperberg, 2013; Fields 
Table 1 Stimulus properties and examples

\begin{tabular}{|c|c|c|c|c|c|c|}
\hline & \multicolumn{3}{|l|}{ Low Arousal } & \multicolumn{3}{|l|}{ High Arousal } \\
\hline & Unpleasant & Neutral & Pleasant & Unpleasant & Neutral & Pleasant \\
\hline Valence & $2.26(0.42)$ & $4.20(0.46)$ & $5.40(0.36)$ & $1.97(0.41)$ & $4.12(0.588)$ & $5.66(0.394)$ \\
\hline Arousal & $3.47(0.43)$ & $3.34(0.38)$ & $3.39(0.45)$ & $4.61(0.56)$ & $4.43(0.491)$ & $4.75(0.490)$ \\
\hline Frequency & $7.81(1.72)$ & $7.98(1.82)$ & $8.23(2.03)$ & $7.91(1.58)$ & $7.88(1.806)$ & $7.86(1.710)$ \\
\hline Concreteness & $3.82(0.91)$ & $4.01(1.07)$ & $3.92(1.15)$ & $3.79(0.95)$ & $3.87(1.073)$ & $3.69(1.000)$ \\
\hline Length & $7.06(2.18)$ & $7.13(1.47)$ & $6.96(1.98)$ & $6.92(1.61)$ & $7.12(1.546)$ & $7.31(1.514)$ \\
\hline Orth & $2.06(2.78)$ & $1.88(3.45)$ & $2.15(3.15)$ & $1.73(3.21)$ & $1.68(2.844)$ & $1.45(2.458)$ \\
\hline Orth_F & $8.44(19.1)$ & $6.13(17.3)$ & $22.90(50.8)$ & $13.80(43.4)$ & $8.26(21.1)$ & $15.85(56.9)$ \\
\hline N2_C & $110.31(89.7)$ & $132.94(93.1)$ & $111.20(93.6)$ & $130.75(108.4)$ & $124.16(99.7)$ & 132.07 (97.5) \\
\hline N2_F & $811.73(617.2)$ & $936.24(678.3)$ & $1,035.03(806.5)$ & $1,010.47(765.7)$ & $884.03(600.9)$ & $1,001.64(919.0)$ \\
\hline \multirow[t]{6}{*}{ Examples } & Stingy & Pacify & Serenity & Atrocity & Splashed & Flourish \\
\hline & Anxiety & Feminine & Loyal & Brutal & Mythical & Delicious \\
\hline & Ignorance & Random & Peace & Hate & Radical & Success \\
\hline & Gangster & Sculpture & Tulips & Tyrant & Spicy & Caressed \\
\hline & Vomit & Apples & Sapphire & Bombs & Samurai & Fireworks \\
\hline & Garbage & Coffee & Food & Murder & Alien & Champion \\
\hline
\end{tabular}

Valence, arousal, and concreteness were all prerated using 7-point Likert scales (from most negative to most positive, least arousing to most arousing, and abstract to concrete, respectively). Frequency was defined as the log of the HAL frequency per million (Balota et al., 2007). Length was defined as the number of letters. The number of orthographic neighbors ("Orth") and the number of word forms that shared the same constrained bigrams ("N2_C") were drawn from the MCWord database (Medler \& Binder, 2005), along with the mean log frequencies of the orthographic neighbors ("Orth F") and the bigrams ("N2_F"). Values are listed as "mean (standard deviation)."

\& Kuperberg, 2012; Holt et al., 2009), but the majority were collected specifically for this study. In all rating studies, our participants (20-50 per rating study) were recruited through online postings. Informed consent was obtained for all participants, who were compensated for their time. Participants completed a guided practice prior to each survey. Then they were asked to rate each word on the specified dimension, on a scale of 1-7. Responses were excluded if participants indicated early language exposure other than English, self-reported psychiatric illness, neurological illness, neurological damage including stroke and concussion, or current treatment with psychoactive medication. In addition, "catch" questions were used to identify and omit bots (computer programs designed to automatically complete paid surveys). Finally, outlying participants were omitted from the ratings, as defined by being an average of two standard deviations or more away from the mean rating for each word.

The final stimulus set included 468 experimental words (159 adjectives, 168 nouns, and 141 verbs; no hyphenated words), 78 per condition ( 3 levels of Valence $\times 2$ levels of Arousal), and 52 animal word fillers (see Table 1 for exemplars). The valence ratings were matched across both levels of arousal, and the arousal ratings were matched across all three levels of valence. In addition, concreteness ratings, HAL log frequency values (Balota et al., 2007), orthographic neighborhood size, and bigram frequency (Medler \& Binder, 2005) were matched across all six conditions. Word class was also matched across conditions, as confirmed by a log-linear analysis of word counts for the valence, arousal, and class categories (all $p \mathrm{~s}>.3$ ). Finally, word length (number of letters) was calculated for each word and was matched across levels of arousal; see Table 1.

The total of 52 animal words were then distributed among the experimental words, comprising $10 \%$ of the total stimulus set of 520. These probe words were matched on word length and HAL frequency to the 468 experimental words (none of which was the name of an animal). Although the animal words were all nouns (as compared to the experimental materials, which were only about one-third nouns), the matched word class across experimental conditions prevented word class from confounding valence and arousal effects, even if participants could use word class as an implicit heuristic to assist in task performance.

\section{ERP study participants}

All participants were recruited through online postings at Tuftslife.com. Data from 26 young adults (13 men, 13 women) were collected. All of the participants were right-handed native English speakers (having learned no other language before the age of 5) between the ages of 18 and 25 . No participants were taking neuropsychiatric medications, and none reported a history of psychiatric or neurological disorders or head trauma. All participants had normal or corrected-tonormal vision. They were compensated for their time 
and provided informed consent in accordance with the procedures of the Institutional Review Board of Tufts University.

\section{Task and experimental procedure}

Each trial started with a "blink sign," written as "( - - )," and began when the participant pressed the "advance" button with the right index finger. After this button had been pressed, a fixation cross appeared at the center of the screen for $1 \mathrm{~s}$ and then disappeared, leaving a blank screen for $500 \mathrm{~ms}$. Then, a word was presented on the screen for 800 ms. Participants were directed to press the "target" button with their right thumb as quickly as possible if the word was identified as an animal word. For all other words, participants were told not to press a button. Each word was followed by $300 \mathrm{~ms}$ of blank screen, followed by a pause at another blink sign.

The full stimulus set was divided into 25 self-paced blocks. Between the blocks, the experiment was paused while "READY" was shown on the screen. Participants were told that they could move their head or hands during this pause only, and that they could continue to the next word by pressing the "advance" button.

\section{Electroencephalography (EEG) recording}

Twenty-nine tin electrodes were held in place on the scalp by an elastic cap (Electro-Cap International, Inc., Eaton, $\mathrm{OH}$ ). Electrodes were also placed below the left eye and at the outer canthus of the right eye to monitor vertical and horizontal eye movements, and on the left and right mastoids. The target impedances were below $5 \mathrm{k} \Omega$ for all scalp and mastoid electrode sites, and below $10 \mathrm{k} \Omega$ for the two eye channels. The EEG signal was collected with a left-mastoid reference and was amplified by an Isolated Bioelectric Amplifier System, Model HandW-32/BA (SA Instrumentation Co., San Diego, CA), with a bandpass of 0.01 to $40 \mathrm{~Hz}$, and was continuously sampled at $200 \mathrm{~Hz}$ by an analog-to-digital converter. The stimuli and behavioral responses were simultaneously monitored by a digitizing computer, and trials were rejected if a blink, head movement, disconnected electrode, missing data, or other artifact was detected between $200 \mathrm{~ms}$ preonset and $800 \mathrm{~ms}$ postonset. The overall artifact rejection rate was $4.76 \%$ (SD $3.91 \%)$. The data were then subject to a $15-\mathrm{Hz}$ low-pass Butterworth filter before analysis, which filtered out frequency domains that were roughly an order of magnitude faster than the a priori amplitude modulations of interest (i.e., the LPC, which is typically averaged over several hundred milliseconds). No additional offline highpass filter was used, due to the possibility that the anticipated LPC modulation could induce illusory early effects (Acunzo, Mackenzie, \& van Rossum, 2012; Rousselet, 2012).

\section{Statistical analysis}

After artifact rejection, the EEG was time-locked to word onset, and the amplitudes were averaged into the six word conditions (see Table 1 for examples). All waveforms were analyzed using a -100 -ms to 0 -ms baseline.

Following several previous studies using both emotional and nonemotional stimuli (Delaney-Busch \& Kuperberg, 2013; Fields \& Kuperberg, 2015; Kreher et al., 2009; Osterhout \& Holcomb, 1992), we adopted the following approach for our systematic statistical analysis: The scalp was subdivided spatially into a number of comparatively shaped regions, each consisting of three electrodes that were averaged together. Because of the strong a priori expectation that the LPC (and other) components would peak near the midline, we first carried out a "mid-regions omnibus analysis of variance" (ANOVA) that included five of these regions arranged down the anterior-posterior axis, along the center of the scalp, shown in dark gray in Fig. 1. This mid-regions omnibus ANOVA included Region (prefrontal, frontal, central, parietal, and occipital), Valence (pleasant, neutral, and unpleasant), and Arousal (high and low) as within-subjects factors. In addition, to extend coverage of the scalp without compromising the simplicity or utility of the a priori mid-regions test, we carried out a "peripheral omnibus ANOVA." This included a second set of four regions along the left and right scalp periphery (shown in light gray in Fig. 1). The within-subjects factors in this second omnibus ANOVA were Anteriority (anterior and posterior), Hemisphere (left and right), Valence (pleasant, neutral, and unpleasant), and Arousal (high and low).

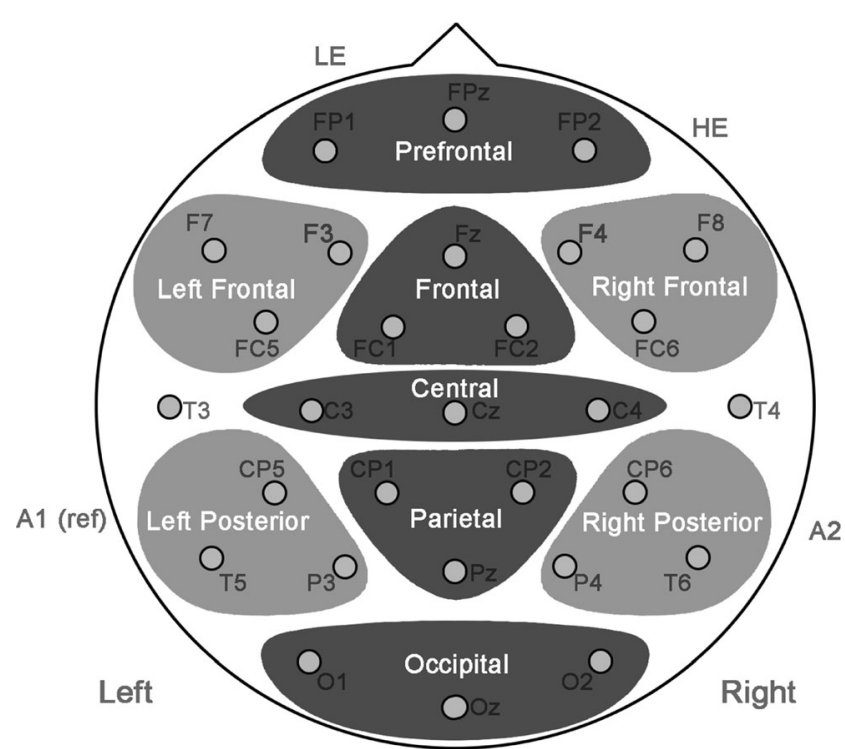

Fig. 1 Scalp regions. For the purposes of our statistical analyses, the scalp was divided into three-electrode regions. The regions in dark gray were part of the mid-regions omnibus ANOVA, and the regions in light gray were part of the peripheral-regions omnibus ANOVA 
For both omnibus tests, significant interactions between valence and arousal were followed up by simple-effects ANOVAs at each level of each experimental variable. Interactions that involved spatial factors (Region, Anteriority, or Hemisphere) were followed up within each level. Significant simple effects of valence were also followed by pairwise effect testing using the Fisher-Hayter method. Alpha was set to .05 for all hypothesis testing, and all effects were corrected using the Greenhouse-Geisser method, where applicable (Greenhouse \& Geisser, 1959).

The results for the LPC time window are reported below. Earlier effects are reported in the supplementary materials.

\section{Results}

\section{Behavioral responses}

Participants' mean accuracy in recognizing the 52 animal words was $94.3 \%(S D=4.8 \%)$, and their mean reaction time was $679 \mathrm{~ms}(S D=66 \mathrm{~ms})$. The mean rate of false alarms (buttonpresses to words that were not animals) was $0.3 \%$. All included participants had accuracy rates higher than $75 \%$, mean reaction times lower than $800 \mathrm{~ms}$, and three or fewer false alarms.

\section{ERP results}

We observed no main effects or two-way interactions for valence and arousal between 0 and $100 \mathrm{~ms}$ or between 100 and $200 \mathrm{~ms}$ in either the mid-regions omnibus ANOVAs (all $p$ s $>$ .3 ) or the peripheral-regions omnibus ANOVAs (all $p \mathrm{~s}>$.1). A P2 component and anterior negativity were apparent in the waveforms, and effects in these time windows are reported in the supplementary materials.

LPC: 500-800 ms A main effect of arousal was observed in the mid-regions omnibus ANOVA $[F(1,23)=8.24, p=.009]$ and approached significance in the peripheral omnibus ANOVA $[F(1,23)=4.23, p=.051]$. This effect was driven by a larger LPC to high- than to low-arousal words. An interaction between arousal and region also reached significance in the mid-regions ANOVA $[F(4,92)=5.52, p=.010]$. To follow up this interaction, we assessed the effect of arousal at each of the mid-regions. The main effect of arousal was maximal over occipital sites $[F(1,23)=14.6, p<.001]$ and remained significant over parietal, central, and frontal sites ( $p \mathrm{~s}<.025)$, but each step toward the anterior decreased the size of the effect (see Fig. 2), until it was no longer significant in the prefrontal mid-region $(p=.495)$. Main effects and interactions involving valence did not reach significance in either omnibus ANOVA (see Fig. 3).

\section{Discussion}

Experiment 1 was designed to determine the effects of valence and arousal during the processing of single emotional words when neither dimension of emotion was relevant to the task at hand. We found a main effect of arousal on the LPC. This effect was independent of valence: The arousal effects on pleasant, unpleasant, and neutral words were statistically indistinguishable in their magnitudes and directions, and qualitatively they appeared to have comparable spatial distributions that were consistent with an LPC effect (see Fig. 2).

The clear effects of arousal on the LPC replicate previous work using lexical-decision and passive-reading tasks (Bayer et al., 2012; Recio et al., 2014). Our findings add to these previous studies by providing evidence that, when emotion was not overtly relevant to task performance, the arousal property of emotional words captured attention and elicited sustained evaluative processing, even when participants were engaged in deep semantic processing. We also found an enhanced P2 to high-arousal (vs. low-arousal) words, regardless of valence (reported in supplementary materials), which might reflect an early orientation of attention to these higharousal words (Crowley \& Colrain, 2004; Kanske, Plitschka, \& Kotz, 2011). A similar pattern of concurrent P2 and LPC arousal effects across posterior electrodes was observed by Bayer et al. (2012) with a lexicaldecision task.

The absence of any effect of valence or any interaction between valence and arousal on the LPC (or the P2) suggests that, with these task demands, valence alone was not sufficient to elicit sustained evaluative processing. This result differs from what has been reported in some previous studies (Bayer et al., 2012; Recio et al., 2014), in which a larger LPC was found to pleasant words than to unpleasant or neutral arousal-matched words. As we noted in the introduction, however, valence may have been implicitly relevant to carrying out the lexical-decision task in these experiments (Kissler \& Herbert, 2013; Kissler \& Koessler, 2011; Kousta et al., 2009; Schacht \& Sommer, 2009).

In sum, these findings suggest that, during deep semantic processing, when emotion is not relevant to task performance, arousal, but not valence, captures attention and leads to sustained evaluative processing. The wider implication of this finding is that, considering the similarities and differences in the previous effects seen using lexical-decision tasks, the relative contributions of valence and arousal to neural processing may depend on the task demands. If this is correct, then valence may trigger sustained evaluative processing if it is made relevant to the task at hand, whereas arousal might have a relatively smaller effect by comparison. Testing this hypothesis was the goal of Experiment 2. 
High Arousal minus Low Arousal: 500-800ms, at each level of Valence

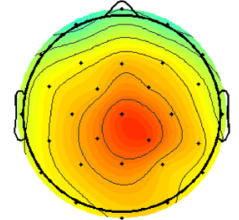

Unpleasant

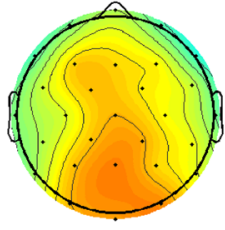

Neutral

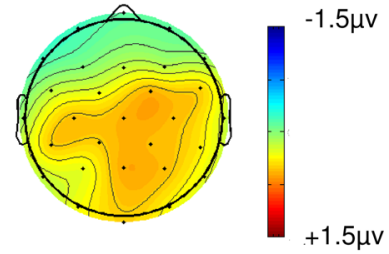

Pleasant
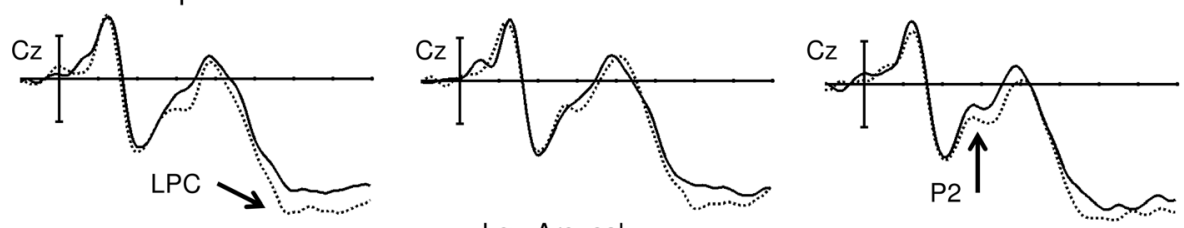

- Low Arousal
….. High Arousal

$-2 \mu \mathrm{v}$

$200 \quad 400 \quad 600 \quad 800 \mathrm{~ms}$

Fig. 2 Experiment 1, semantic-monitoring task: Effect of arousal at each level of valence. Unpleasant, neutral, and pleasant words yielded arousal effects that did not statistically differ in amplitude (as can be seen with the

\section{Experiment 2}

In Experiment 2, the same stimulus materials were shown to a new group of participants. This time, participants were asked to overtly evaluate the valence of each word, designating them as "pleasant," "unpleasant," or "neutral" in a forced choice categorization task (with no time pressure). On the basis of previous research demonstrating that ERP emotion effects on the LPC are largest when attention is overtly oriented toward valence (Fischler \& Bradley, 2006), we expected to find a larger LPC to high-arousal valenced words (in general) than to low-arousal neutral words. Our question pertained to how each particular dimension of emotion contributes to this effect. We considered three possibilities.

The first was that, as for Experiment 1, the LPC would be driven primarily by arousal. This would imply that sustained evaluative processing is elicited by the attention-grabbing

Valence Effects for High Arousal words: $500-800 \mathrm{~ms}$

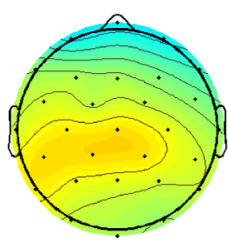

Unpleasant minus Neutral

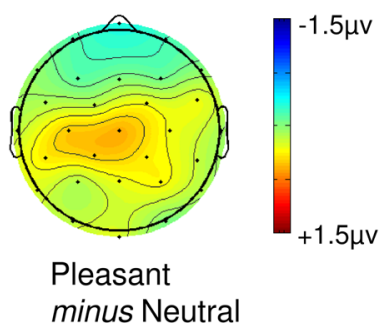

waveforms shown for electrode $\mathrm{Cz}$ ) and that appeared to show comparable distributions (as is shown with the voltage maps)

high-arousal words in a relatively fixed manner, irrespective of task requirements. The second was that the LPC would be driven by a combination of valence and arousal. For example, the effect of arousal on the LPC might be larger for unpleasant and pleasant words than for neutral words. This would suggest that, when valence is task-relevant, valence and arousal contribute in tandem to sustained evaluative processing.

The third possibility was that, with this explicit valence evaluation task, the LPC would be driven predominantly by valence instead of arousal. This could appear as a negativity bias (Ito et al., 1998), a positivity bias (Bayer et al., 2012), or an overall advantage for all valenced words (vs. neutral words). This inversion of the main effects found in Experiment 1, using the same stimuli, would provide strong evidence that the relative contributions of valence and arousal to the LPC are, in part, determined by the demands of a given situation, and are not fixed consequences of the words themselves.

Valence Effects for Low Arousal words: $500-800 \mathrm{~ms}$

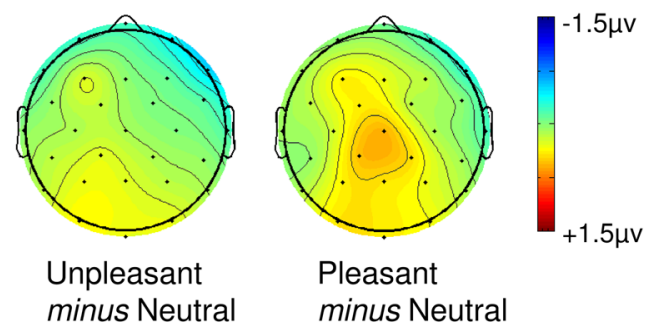

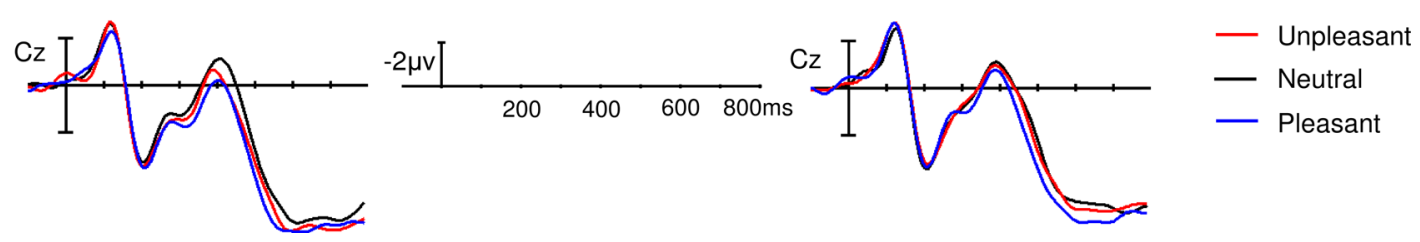

Fig. 3 Experiment 1, semantic-monitoring task: Effect of valence at each level of arousal. No valence effects reached significance on the LPC (500-800 ms) 


\section{Method}

The same 468 experimental words were used in a 3 (Valence: pleasant, unpleasant, neutral) $\times 2$ (Arousal: high, low) experimental design, but the 52 animal words from Experiment 1 were omitted. Data were initially collected from 26 young adults (13 men, 13 women), but four of the participants were rejected because of excessive artifacts, leaving 22 participants (11 men, 11 women; mean age 20.3 years). The procedures were the same as in Experiment 1, except that participants were directed to classify each word as "positive, " "neutral," or "negative." Participants made their responses following a "?" cue, which appeared after the 800-ms word presentations (following an interstimulus interval of $300 \mathrm{~ms}$ ), with no time pressure. All responses were made using the right thumb, with the "negative" button slightly to the left of center, "neutral" in the middle, and the "positive" button slightly to the right (to correspond with the intuitions of right-handed participants and reduce errors).

Data processing and statistical analysis of the ERP data were the same as in Experiment 1. ERPs were averaged according to the original categorizations (pleasant, neutral, and unpleasant) from the ratings studies, in order to retain the careful counterbalancing. However, we also report a post-hoc analysis of ERPs averaged by the idiosyncratic behavioral classifications of each individual participant. The average artifact rejection rate was $5.06 \%$ per participant $(S D=4.10 \%)$.

\section{Results}

\section{ERP results}

We found no main effects or two-way interactions of valence and arousal between 0 and $100 \mathrm{~ms}$ or between 100 and $200 \mathrm{~ms}$ in either the mid-regions omnibus ANOVAs (all $p \mathrm{~s}>.3$ ) or the peripheral-regions omnibus ANOVAs (all $p s>$.1). A P2 component and anterior negativity were apparent in the waveforms, and the effects within these time windows are reported in the supplementary materials. Arousal effects are shown in Fig. 4 and valence effects are shown in Fig. 5.

LPC: 500-800 ms A main effect of valence was significant in both the mid-regions $[F(2,42)=4.62, p=.015]$ and the peripheral-regions $[F(2,42)=5.73, p=.006]$ omnibus ANOVAs. The magnitude of this valence effect varied across the scalp, reflected by interactions between valence and region in the mid-regions ANOVA $[F(8,168)=3.42, p=.016]$, and between valence and anteriority in the peripheral-regions ANOVA $[F(2,42)=6.64, p=.003]$. Follow-up ANOVAs revealed significant effects of valence only in central, parietal (mid and lateral), and occipital regions ( $p s<.01)$, due to a larger LPC on unpleasant words than on either pleasant (all $p \mathrm{~s}<.05$ ) or neutral (all $p \mathrm{~s}<.01$ ) words (see Fig. 4). We observed no significant differences between pleasant and neutral words in any of these regions. Main effects and interactions involving arousal were not significant in either omnibus ANOVA (see Fig. 5).

LPC to each participant's valence judgments We also assessed the valence effects on the ERPs elicited by words individually classified as "positive," "negative," or "neutral, " regardless of the prerated categories (see Fig. 6). This analysis reflected a test of "perceived valence category" on LPC amplitudes, with the caveat that each participant had a different number of trials per condition, and the careful counterbalancing of potentially confounding factors between conditions was no longer maintained. In addition to main effects of valence [mid-region: $F(2,42)=7.85, p$ $=.002$; peripheral: $F(2,42)=7.94, p=.002]$, we observed an interaction between valence and region in the mid-regions ANOVA $[F(8,168)=5.36, p<.001]$ that was echoed by an interaction between valence and anteriority in the peripheral regions ANOVA $[F(2,42)=9.78$, $p<.001]$. Follow-ups showed that valence effects were maximal over posterior regions (with parietal, occipital, and posterior peripheral regions showing the most significant effects, with $p s<.001)$. Here, however, the pattern was somewhat different from that described above. Once again, unpleasant words evoked a larger LPC than did neutral words (all $p \mathrm{~s}<.001$ ), but as is shown in Fig. 6, pleasant words also evoked a larger LPC amplitude than did neutral words (all $p \mathrm{~s}<.05$ ), and unpleasant words evoked a larger LPC than pleasant words only over the posterior periphery $(p=.007)$.

\section{Relationship between participants' valence judgments} and ERP data to the prerated valence categories The correspondences between each participant's own valence categorizations during the ERP experiment and our prior classifications of the three emotional conditions (using the norming data described under Exp. 1) are shown in Table 2a. As can be seen there, participants tended to misclassify neutral words more often than emotional words. The breakdown of classifications indicates that misclassified neutral words were most likely to be misclassified as "positive" (median 54.0 out of the 156 neutral words) rather than as "negative" (median 17.5 out of the 156 neutral words).

To quantify the extent to which participants tended to discriminate unpleasant and pleasant words from the neutral words, we carried out a $d^{\prime}$ analysis (see Table $2 \mathrm{~b}$ ). This confirmed that the participants' ability to discriminate unpleasant and neutral words was significantly better than their ability to discriminate pleasant and neutral words $(t=6.12, p<.001$, for high-arousal words; $t=15.04, p<.001$, for low-arousal words). 
High Arousal minus Low Arousal: $500-800 \mathrm{~ms}$, at each level of Valence

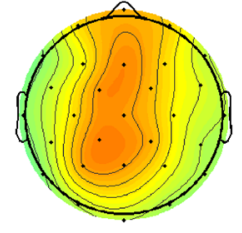

Unpleasant

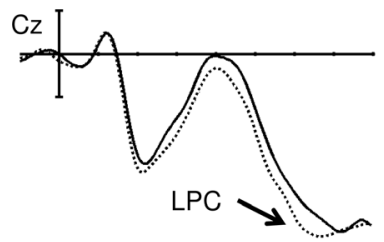

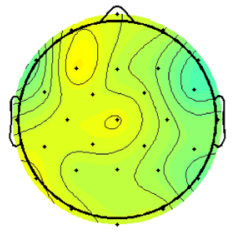

Neutral

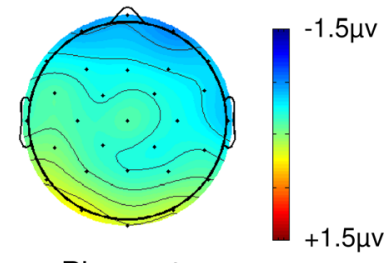

Pleasant
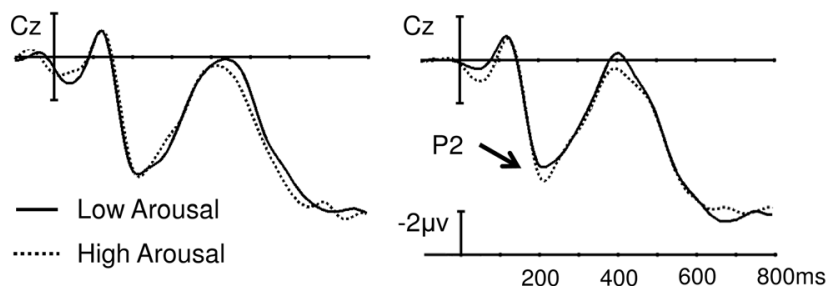

Fig. 4 Experiment 2, valence judgment task: Effect of arousal at each level of valence. No arousal effects reached significance on the LPC (500-800 ms)

Finally, to directly test the hypothesis that a participant's ability to discriminate pleasant or unpleasant words from neutral words was related the size of the LPC effect for that person, we calculated a Pearson's correlation coefficient between each participant's $d^{\prime}$ scores (averaged across high- and lowarousal words) and the amplitude of each participant's pairwise valence effects (both unpleasant vs. neutral and pleasant vs. neutral) in each of the three regions where the LPC was largest (the central, parietal, and occipital mid-regions). We found that the more discriminable the unpleasant words were from the neutral words (i.e., the larger the $d^{\prime}$ score), the larger was the LPC effect to unpleasant (vs. neutral) words over the occipital mid-region $\left(r^{2}=.198, p=.038\right)$. Similarly, the more discriminable the pleasant words were from the neutral words (i.e., the larger the $d^{\prime}$ score), the larger the LPC effect to pleasant (vs. neutral) words over the central $\left(r^{2}=.224, p=.026\right)$ and parietal $\left(r^{2}=.223, p=.027\right)$ mid-regions. In other words, the size of the LPC valence effect was directly related to the perceived distinction between the prerated valence categories (as measured by overt valence judgments).

Between-subjects task effects To overtly test the influence of experimental task on the processing of valence and arousal, we conducted a group-level ANOVA, with Task as a between-subjects factor and Valence and Arousal as within-subjects factors. To limit unacceptable inflation of the familywise error rate, we restricted our analysis to a single test at the parietal mid-region, where the valence and arousal effects on the LPC tend to be maximal (Citron, 2012), as we had found in the present experiments as well.

The combined data revealed an interaction between task and valence $[F(2,88)=3.88, p=.026]$, indicating that the valence effects differed significantly between experiments.
Valence Effects for High Arousal words: $500-800 \mathrm{~ms}$

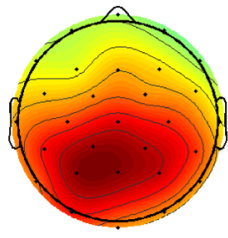

Unpleasant minus Neutral

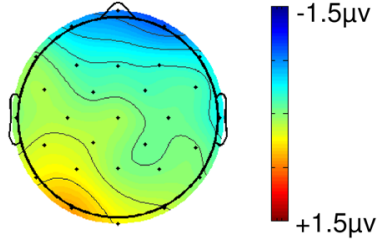

Pleasant minus Neutral
Valence Effects for Low Arousal words: $500-800 \mathrm{~ms}$
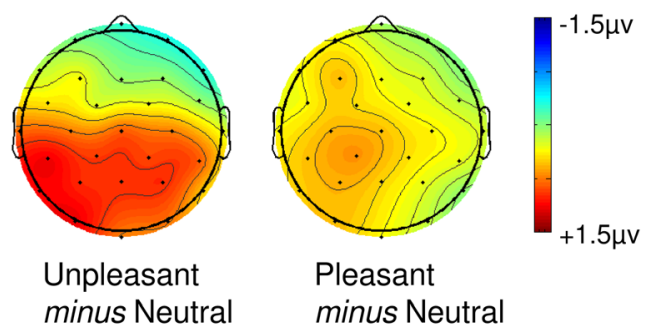

- Unpleasant

- Neutral

- Pleasant
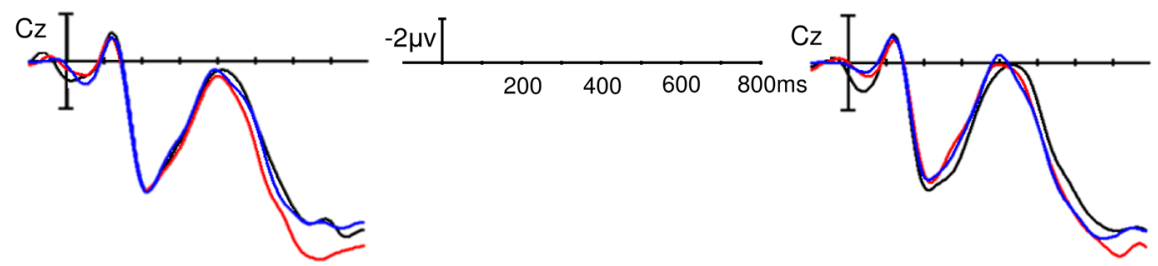

Fig. 5 Experiment 2, valence judgment task: Effect of valence at each level of arousal. Over central-posterior electrodes, unpleasant words elicited a larger LPC than did neutral and pleasant words. These valence effects did not significantly differ between the high- and low-arousal words 


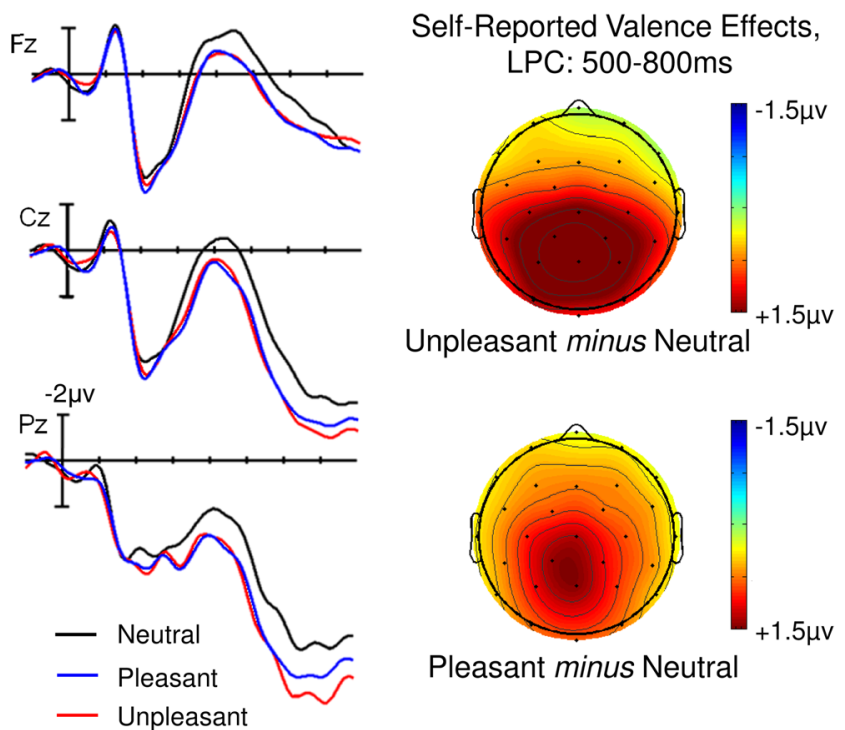

Fig. 6 Experiment 2, effects of self-reported valence. Words that participants categorized as pleasant or unpleasant elicited larger LPCs than did words that participants categorized as neutral

However, the task by arousal interaction did not reach significance $[F(1,44)=2.66, p=.110]$. The three-way interaction between task, valence, and arousal was also nonsignificant $[F(2,88)=0.55, p=.577]$. As expected, we observed significant within-subjects main effects of both valence $[F(2,88)=7.15, p=.002]$ and arousal $[F(1,44)=7.75, p=.008]$, with no interaction between the two $[F(2,88)=1.98, p=.146]$.

Table 2 Experiment 2 valence categorizations

(a) Median Numbers of Valence Categorizations Across Participants

$\begin{array}{llll}\begin{array}{l}\text { Prerated Valence } \\ \text { Category }\end{array} & \text { "Positive" } & \text { "Neutral" } & \text { "Negative" } \\ \text { Pleasant } & 127 & 23.5 & 2 \\ \text { Neutral } & 54 & 79.5 & 17.5 \\ \text { Unpleasant } & 2 & 11 & 140.5\end{array}$

(b) $d^{\prime}$ Scores for Discriminating Valenced Words From Neutral Words Pleasant From Unpleasant From Neutral Neutral

High arousal $\quad 1.68(0.38) \quad 2.44(0.48)$

$\begin{array}{lll}\text { Low arousal } & 1.21(0.23) & 2.63(0.46)\end{array}$

In all, 156 words from each of the prerated valence categories (leftmost column in section a) were shown to participants, but some were classified by participants as a different valence from the prerated norms. Because the extent of deviation from the prerated norms had a skewed distribution, the median number of categorizations is shown in Table $2 a$, instead of the mean. A false positive was defined as a valence judgment that did not coincide with the prerated norms. A $d^{\prime}$ score (shown here in section b) was calculated for each person at each level of arousal for both the pleasant-versus-neutral and the unpleasant-versus-neutral boundary (omitting data from the third valence category in each case). Values are listed as "mean (standard deviation)"

\section{Discussion}

In Experiment 2 we aimed to determine whether valence could influence sustained evaluative processing of the same emotional words that previously had elicited only an arousal effect under different task demands. We found this to be the case. There was clear main effects of valence on the LPC, which were statistically indistinguishable between high-arousal and low-arousal words (Fig. 5). This main effect of valence was driven by a larger LPC on unpleasant words, relative to both neutral and pleasant words, over the regions that typically capture the LPC (Hajcak et al., 2010; Hajcak et al., 2012).

The larger LPC amplitude to unpleasant than to neutral words is consistent with the results of most previous studies (Citron, 2012; Kissler et al., 2006). Here, we extended this result to words matched at both high and low levels of arousal. The larger LPC amplitude to unpleasant than to pleasant words is also consistent with several previous ERP studies, with both words (Delaney-Busch \& Kuperberg, 2013; Fields \& Kuperberg, 2012; Holt et al., 2009) and pictures (Ito et al., 1998), across a variety of valence evaluation and comprehension tasks. This effect has been interpreted as a neural correlate of an inherent attentional bias toward negative stimuli-a "negativity bias" (Carretié, 2014; Carretié, Albert, López-Martin, \& Tapia, 2009; Ito et al., 1998). Although the finding of a negativity bias for the LPC in general is not novel, the present ERP study is, to our knowledge, the first to show a negativity bias for low-arousal words. Specifically, the LPC was larger to low-arousal unpleasant words like "moldy" than to low-arousal pleasant words like "bouquet."

As one might expect, the way that any particular individual classified the valence of a given word during the ERP experiment did not necessarily mirror our prior ratings-based categorizations of the words. Given that our prior categorizations were based on average ratings, this is not surprising: There is bound to be some variability in how different individuals perceive the valences of different words. For example, although "fireworks" - a word that fell in the pleasant category, in terms of our prior ratings - might be perceived as pleasant by most people, some people might be indifferent to fireworks and perceive the word as neutral, and others may perceive fireworks as being startling and altogether unpleasant. Similarly, we observed significant natural variation in where individuals implicitly defined the boundaries between the valence categories while making their judgments. For instance, some participants tended to classify a relatively large proportion of the words as pleasant or unpleasant (indicating that most stimuli were perceived as highly valenced), whereas other participants tended to classify a relatively small proportion of words as pleasant or unpleasant (indicating that significantly fewer stimuli were perceived as highly valenced). Finally, some discrepancies 
between our prior ratings-based categorizations and participants' classifications during the ERP experiment may have arisen as a result of differences in the task requirements (rating on a Likert scale of 1-7 vs. classifying into one of three nominal categories during the ERP experiment) and broader experimental contexts (e.g., differences in the natures of the surrounding words).

Examination of the pattern of participants' average behavioral classifications during the ERP experiment revealed some insights into the nature of the negativity bias. Our results showed that, whereas participants tended to classify the unpleasant words in a way that was consistent with our prior ratings-based categorizations, this was less true of the pleasant words, which were often categorized as neutral (see Table 2a). These data therefore raise the possibility that the larger LPC to unpleasant than to neutral words - the negativity bias, which was also based on average data - was driven by the relatively clear and universal distinction between unpleasant and neutral words (vs. between pleasant and neutral words). This suggests that words clearly perceived as pleasant by particular participants may also generally elicit larger LPC amplitudes than words perceived as neutral, regardless of the words' prerated norms. Examination of the same ERP data categorized by participant's subjective valence classifications of each word during the experiment itself (rather than by our prior ratingsbased categorizations) supported this interpretation: The negativity bias disappeared. Instead, the amplitudes of the LPC were larger to both unpleasant and pleasant words than to neutral words, with no difference between pleasant and unpleasant words at most sites (Fig. 6).

This result carries implications for the interpretation of the negativity bias effect on the LPC. Classically, the larger LPC to unpleasant than to pleasant arousal-matched words has been interpreted as reflecting the "tendency for the negative motivational system to respond more intensely than the positive motivational system to comparable amounts of activation" (Ito et al., 1998, p. 888)-a difference in scale among otherwise equivalent valence effects (Rozin \& Royzman, 2001; Taylor, 1991). Our data suggest an alternative contribution: that the negativity bias could reflect the extent to which unpleasant words are more distinct or discriminable (from neutral words) than pleasant words, given the particulars of the context and the demands of the situation.

More generally, these findings also have important implications for the understanding the functional significance of the emotional LPC: They suggest that the LPC might be primarily sensitive to individual participants' perceived, subjective valences. In this study, given the requirement to categorize the words into one of three valence groups, this proxied for each individual's propensity to discriminate valenced words (both pleasant and unpleasant) from the neutral words. In support of this interpretation, we found that $d^{\prime}$ scores, reflecting each individual's ability to discriminate unpleasant from neutral words, as well as to discriminate pleasant from neutral words, correlated with the magnitudes of the LPC effect to both unpleasant and pleasant (vs. neutral) words, respectively.

In sum, the valence task led to a robust valence effect, in which the LPC may have reflected subjective perceived valence. Taken together with the data from Experiment 1, these data indicate that the task can heavily influence the processing of emotional words. We turn to the functional significance of these differences between the two experiments next in the General Discussion.

\section{General discussion}

This study had two related aims: The first was to determine how valence and arousal contribute to the neural processes engaged during the online evaluation of emotional words, using a design that fully crossed two levels of arousal (high and low) and three levels of valence (pleasant, unpleasant, and neutral) in a large, carefully controlled sample of words (see also Bayer et al., 2012; Recio et al., 2014). The second was to determine how these effects of emotional properties are influenced by their task relevance. Our findings were clear. We showed that valence and arousal both can elicit an effect on the LPC, but that the particular pattern of their respective contributions depends significantly on task. In Experiment 1, when participants performed a semantic categorization task in which emotion was not relevant to task performance, the LPC showed a large effect of arousal (Fig. 2), but no effect of valence (Fig. 3). In contrast, in Experiment 2, in which participants performed an overt valence categorization task, the LPC showed a large effect of valence (Fig. 5), but no effect of arousal (Fig. 4).

These data are fully consistent with the large body of previous research reporting overall "emotion" effects to words on the LPC under a broad array of experimental conditions (Citron, 2012; Kissler et al., 2006): In both Experiment 1 and Experiment 2, high-arousal valenced words elicited larger LPC amplitudes than did low-arousal neutral words. The results of Experiment 1 are also consistent with previous research reporting effects of arousal within a single level of valence (Bayer et al., 2012; Delplanque et al., 2006; Recio et al., 2014), though we extended these previous findings to a novel task, and to neutral words that also showed an arousal effect comparable to the pleasant and unpleasant words. The results of Experiment 2 are consistent with research reporting LPC effects of valence within a single level of arousal (Bayer et al., 2012; Hajcak \& Olvet, 2008; Ito et al., 1998; Recio et al., 2014), though we extended these previous findings to a novel task and established that a negativity bias can be elicited using both high- and low-arousal words. Finally, like previous research (Bayer et al., 2012; Recio et al., 2014), we found no interaction effect between valence and arousal on the LPCs in 
either experiment, and we extended the previous work on the independence of these two factors during word processing to two new tasks.

What is most novel about our findings is that we showed, for the first time, that changes in task can lead to robust changes in the relative contributions of valence and arousal to the LPC. Previous ERP studies have reported that "emotion" effects on the LPCs elicited by emotional (vs. neutral) words (Fischler \& Bradley, 2006) and pictures (Dunning \& Hajcak, 2009; Hajcak et al., 2009; Hajcak et al., 2010; Olofsson et al., 2008) can grow or shrink depending on the task demands. Other work, such as that by Bayer et al. (2012), has reported effects of both valence and arousal on the LPC during a lexical-decision task (see also Recio et al.) and a passivereading task, suggesting that valence and arousal may be processed independently during reading. In this study, however, we showed that a change in task relevance can completely invert the observed pattern of effects: When emotion was task-irrelevant, only an arousal effect manifested on the LPC, but when valence was overtly relevant, only a valence effect manifested on the LPC. A between-subjects analysis additionally indicated that the valence effect may have been the primary contributor to this difference in outcomes, since the valence effect was significantly larger in the valence task than in the semantic task, whereas the apparent complementary change in the arousal effect was not distinguishable from chance (meaning that despite the difference in the overall patterns of results, we cannot conclude that arousal effects in semantic categorization tasks are generally larger than arousal effects in valence judgment tasks).

We attribute these differences in ERP modulation across the two experiments to differences in the relative relevances of valence and arousal to task performance. In Experiment 1, participants needed to make a decision about each word on the basis of its semantic features (whether or not the word was an animal word), so the emotional features of each word (both their valence and arousal) were essentially a distraction from effectively carrying out this task. Under these task conditions, high-arousal words seemed to engage attention (as indicated by the P2; see the supplementary materials) and to elicit sustained evaluative processing (as indicated by the LPC), consistent with the known impact of arousal in lure and distraction paradigms (Mather \& Sutherland, 2011). In contrast, in Experiment 2, participants made decisions about each word on the basis of its valence, and valenced words elicited more sustained evaluative processing (as reflected by the LPC) than did neutral words. The arousal effects on the LPC (and the P2), however, disappeared entirely, possibly because every emotional word was already attended and overtly evaluated as part of the task demands.

However, it is possible that other differences between the two tasks contributed to the different patterns of effects, such as differences in their requirements for overt motor responses.
Specifically, in Experiment 1, although participants made semantic decisions on each word, they were not required to make overt motor responses on the experimental items themselves (these were essentially no-go trials). In Experiment 2, however, the participants were required to make motor responses on all items. However, this alone is unlikely to have driven the difference in ERP findings. Although the P300 family of components (including the LPC) is thought to be related to decision-making and the identification of salient targets (as reviewed by Pritchard, 1981, and Twomey, Murphy, Kelly, \& O'Connell, 2015), it does not specifically reflect motor response demands or response selection (Mccarthy \& Donchin, 1981). Furthermore, in Experiment 2 we introduced a forced delay before buttonpresses on the experimental items, in order to reduce any motor contamination of the ERP responses. It is possible, however, that this forced delay introduced a second task-related difference: In Experiment 1, participants were under more time pressure to make their decisions than in Experiment 2. It is possible that this requirement for a speeded response contributed to the selective effect of arousal in Experiment 1 versus 2, by emphasizing haste (whereas Exp. 2 emphasized more deliberate evaluations).

\section{Neural implications}

These findings have two important neural implications. First, they indicate that the dimensions of valence and arousal can act independently to influence the online neurocognitive evaluation of emotional words. The fact that valence and arousal each modulated similar LPC components, which qualitatively showed similar time courses and topographies across the two experiments, suggests that each dimension may have independently influenced the recruitment of a common neurocognitive mechanism, such as a general reevaluation or reanalysis of the eliciting stimulus in relation to its context (context updating), as has been hypothesized for the related P300 component (Donchin 1981; Donchin \& Coles, 1988). Of course, it is possible that distinct neuroanatomical networks contributed to the effect of arousal in Experiment 1 and the effect of valence in Experiment 2: Because the spatial resolution of ERPs is poor and multiple sources are likely to contribute to the emotional LPC (Foti, Hajcak, \& Dien, 2009), this study alone cannot address this question.

Second, these findings show that the neurocognitive processes elicited by emotional words are not fixed. Rather, we seem to engage in quite different modes of processing to identical sets of words, depending on the task demands (see Lai et al., 2012, for behavioral evidence of such a malleable and dynamic emotion evaluation system). Specifically, when emotion was not overtly relevant to the task demands, as in Experiment 1, highly arousing stimuli triggered a sustained 
emotion evaluation from the bottom up (as indexed by the LPC). However, in situations in which emotion was relevant to the task demands, sustained evaluative processing was driven primarily by valence, regardless of arousal. This illustrates a subtle but critical theoretical point: The dimensions of emotional significance are not strictly a property of the eliciting stimulus, but rather reflect the relationship between the stimulus, the perceiver, and the context (Okon-Singer et al., 2013).

\section{Theoretical implications}

Our findings also have implications for theoretical models discussing how the various dimensions of emotional stimuli influence the processing of emotional words. We suggest that the findings are generally consistent with the circumplex model of the structure of affect, which proposes that the bipolar dimensions of valence and arousal are separate and orthogonal, and that each uniquely contributes to aspects of our evaluations of emotional stimuli (Bradley \& Lang, 2007; Osgood et al., 1967; Russell, 1980). Although the circumplex model was initially derived from explicit behavioral semantic evaluations, our data show that these dimensions seem to correspond to online neural processing, as well, albeit in a dynamic fashion. In particular, our finding in Experiment 1 of a clear effect of arousal even for neutral items (see also Bayer et al., 2012, and Recio et al., 2014) shows that a stimulus does not need to be clearly pleasant or unpleasant for it to lead to prolonged neural processing. It is sufficient for the stimulus simply to be arousing.

On the other hand, we think it likely that "neutral" stimuli are never actually completely devoid of appetitive or aversive significance (Lebrecht, Bar, Barrett, \& Tarr, 2012). Instead, we see "neutral valence" as a useful category for stimuli with very low motivational significance, or "microvalences" (Lebrecht et al., 2012). As such, high-arousal neutral words like "alien" and "rouse" could elicit sustained evaluative processing in a manner similar to high-arousal pleasant and unpleasant words. The activation (arousal) levels are comparable between the words, but the motivational significance (valence) of the neutral words is smaller (though never completely nonexistent).

In contrast, our data are less consistent with models (such as the evaluative space model, or ESM) that have expressed an affect structure in which valence is integrated with arousal during emotion processing (Norris, Gollan, Berntson, \& Cacioppo, 2010). These models propose that arousal increases as a natural consequence of increases in pleasantness or unpleasantness. Although this model of affective structure parsimoniously describes much of human and animal behavior (e.g., action preparedness often seems to require a direction of motivation - see the reviews by Cacioppo \& Bernston, 1994; Norris et al., 2010), it would have predicted correlated effects of valence and arousal during word processing in the present study, for which we found no evidence. On the other hand, although we have provided evidence against the integration of valence and arousal expressed in the most recently articulated versions of the ESM, the present study does not address whether the structure of valence alone is bipolar (ranging from pleasant to unpleasant) or unipolar (ranging both from neutral to pleasant and from neutral to unpleasant), which is the much more central hypothesis of the ESM. It remains possible that unipolar "positivity" and "negativity" dimensions remain orthogonal to a distinct arousal dimension during the processing of emotional words.

Finally, these data are also somewhat inconsistent with other interactive accounts of valence and arousal, which have argued that low levels of arousal or pleasant stimuli trigger approach motivations, whereas high levels of arousal or unpleasant stimuli trigger avoidance motivations (Robinson, Storbeck, Meier, $\&$ Kirkeby, 2004). If it manifested during word processing, this account might predict that high-arousal pleasant stimuli and low-arousal unpleasant stimuli would elicit larger LPCs than low-arousal pleasant or high-arousal unpleasant stimuli, because the motivational signals would be in conflict and require extended consideration to resolve the ambiguity. Although some studies have shown evidence of this interaction (Citron et al., 2013; Feng et al., 2014), the large studies by Recio et al. (2014) and Bayer et al. (2012) provided contrary evidence using lexical-decision and passive-reading tasks. The present study now extends this pattern (of no interactions) to semantic categorization and valence evaluation tasks.

\section{Open questions}

Our results and interpretation raise important questions for future research. Particularly, given the apparent malleability of evaluative processing, it will be important to look beyond the role of task instructions to the effects of other aspects of context on the LPC. There is already strong behavioral evidence that local context can dramatically influence emotional word processing (Lai et al., 2012), and recent ERP experiments have shown that the processing of emotional words is influenced by preceding single words (Delaney-Busch, 2013; Herring, Taylor, White, \& Crites, 2011), sentence contexts (Ding, Wang, \& Yang, 2015; Holt et al., 2009), and discourse contexts (Delaney-Busch \& Kuperberg, 2013; Moreno \& Vázquez, 2011), with similar findings for pictures (Foti \& Hajcak, 2008). It is also possible that the wider structure of an experimental environment can influence how the LPC is modulated. For example, in a recent study we manipulated the presence or absence of taboo words within the wider experimental context (Fogel, Midgley, Delaney-Busch, \& Holcomb, 2013; the manuscript is currently in preparation), finding attenuated LPC emotion effects when the surrounding stimuli were more extreme. The effects of local and broad context, manner of presentation, and individual differences 
between participants remain important and understudied avenues for future research (Kuppens, Tuerlinckx, Russell, \& Barrett, 2013).

\section{Conclusions}

Our results were clear. When emotion was irrelevant to task performance (Exp. 1), the high-arousal words elicited a larger LPC than did low-arousal words, indicating a sustained evaluation of emotional and motivational significance, regardless of valence. When valence was overtly relevant to the given task (Exp. 2), unpleasant words elicited a greater LPC than did neutral words, regardless of level of arousal (and words perceived by individuals as pleasant or unpleasant elicited a larger late positivity than did words perceived as neutral). As a whole, these data suggest that valence and arousal act independently to influence word processing, and that these dimensions of emotion are not simply useful mathematical derivations of meaning, but that they actually reflect how emotional words are processed by the brain. Above all, these experiments illustrate the importance of task: The evaluative systems underlying the LPC not only wax and wane along with the relevance of emotion in general, but also mediate the relative contributions of valence and arousal to word processing in tune with situational demands.

Author note This study was funded by the National Institute of Mental Health (Grant No. R01MH071635 to G.R.K.) and by the Sidney Baer Trust. We thank several people who contributed to constructing the experimental materials, to data collection, and to technical support, including Arim Choi, Allison Fogel, Vivian Haime, Ju Hyung Kim, and Ann Yacoubian. We also thank Marianna Eddy, Eric Fields, Phil Holcomb, Ellen Lau, Katherine Midgley, Heather Urry, and three gracious anonymous reviewers for their insightful comments and technical guidance.

\section{References}

Abelson, R. P., \& Sermat, V. (1962). Multidimensional scaling of facial expressions. Journal of Experimental Psychology, 63, 546-554. doi: 10.1037/h0042280

Acunzo, D. J., Mackenzie, G., \& van Rossum, M. C. (2012). Systematic biases in early ERP and ERF components as a result of high-pass filtering. Journal of Neuroscience Methods, 209, 212-218. doi:10. 1016/j.jneumeth.2012.06.011

Anderson, A. K. (2005). Affective influences on the attentional dynamics supporting awareness. Journal of Experimental Psychology. General, 134, 258-281. doi:10.1037/0096-3445.134.2.258

Aquino, J. M., \& Arnell, K. M. (2007). Attention and the processing of emotional words: Dissociating effects of arousal. Psychonomic Bulletin \& Review, 14, 430-435. doi:10.3758/BF03194084

Arnell, K. M., Killman, K. V., \& Fijavz, D. (2007). Blinded by emotion: Target misses follow attention capture by arousing distractors in RSVP. Emotion, 7, 465-477. doi:10.1037/1528-3542.7.3.465

Balota, D. A., Yap, M. J., Cortese, M. J., Hutchison, K. A., Kessler, B., Loftis, B., . . . Treiman, R. (2007). The English Lexicon Project. Behavior Research Methods, 39, 445-459. doi:10.3758/BF03193014
Baumeister, R. F., Bratslavsky, E., Finkenauer, C., \& Vohs, K. D. (2001). Bad is stronger than good. Review of General Psychology, 5, 323370. doi:10.1037/1089-2680.5.4.323

Bayer, M., Sommer, W., \& Schacht, A. (2010). Reading emotional words within sentences: The impact of arousal and valence on eventrelated potentials. International Journal of Psychophysiology, 78, 299-307. doi:10.1016/j.ijpsycho.2010.09.004

Bayer, M., Sommer, W., \& Schacht, A. (2011). Emotional words impact the mind but not the body: Evidence from pupillary responses. Psychophysiology, 48, 1554-1562. doi:10.1111/j.1469-8986.2011. 01219.x

Bayer, M., Sommer, W., \& Schacht, A. (2012). P1 and beyond: Functional separation of multiple emotion effects in word recognition. Psychophysiology, 49, 959-969. doi:10.1111/j.1469-8986. 2012.01381.x

Bernat, E., Bunce, S., \& Shevrin, H. (2001). Event-related brain potentials differentiate positive and negative mood adjectives during both supraliminal and subliminal visual processing. International Journal of Psychophysiology, 42, 11-34. doi:10.1016/S01678760(01)00133-7

Bradley, M. M., \& Lang, P. J. (1999). Affective norms for English words (ANEW): Stimuli, instruction manual and affective ratings (Technical Report No. C-1). Gainesville, FL: University of Florida, Center for Research in Psychophysiology.

Bradley, M. M., \& Lang, P. J. (2007). Emotion and motivation. In J. T. Cacioppo, L. G. Tassinary, \& G. G. Berntson (Eds.), Handbook of psychophysiology (3rd ed., pp. 581-607). Cambridge: Cambridge University Press.

Briesemeister, B. B., Kuchinke, L., \& Jacobs, A. M. (2014). Emotion word recognition: Discrete information effects first, continuous later? Brain Research, 1564, 62-71. doi:10.1016/j.brainres.2014.03.045

Briggs, K. E., \& Martin, F. H. (2009). Affective picture processing and motivational relevance: Arousal and valence effects on ERPs in an oddball task. International Journal of Psychophysiology, 72, 299 306. doi:10.1016/j.ijpsycho.2009.01.009

Cacioppo, J. T., \& Bernston, G. G. (1994). Relationship between attitudes and evaluative space: A critical review, with emphasis on the separability of positive and negative substrates. Psychological Bulletin, 115, 401-423. doi:10.1037/0033-2909.115.3.401

Carretié, L. (2014). Exogenous (automatic) attention to emotional stimuli: A review. Cognitive, Affective, \& Behavioral Neuroscience, 14, 1228-1258. doi:10.3758/s13415-014-0270-2

Carretié, L., Albert, J., López-Martin, S., \& Tapia, M. (2009). Negative brain: An integrative review on the neural processes activated by unpleasant stimuli. International Journal of Psychophysiology, 71, 57-63. doi:10.1016/j.ijpsycho.2008.07.006

Carretié, L., Hinojosa, J. A., Albert, J., López-Martin, S., de la Gándara, B., Igoa, J. M., \& Sotillo, M. (2008). Modulation of ongoing cognitive processes by emotionally intense words. Psychophysiology, 45, 188-196. doi:10.1111/j.1469-8986.2007.00617.x

Citron, F. M. (2012). Neural correlates of written emotion word processing: A review of recent electrophysiological and hemodynamic neuroimaging studies. Brain and Language, 122, 211-226. doi:10. 1016/j.bandl.2011.12.007

Citron, F. M., Weekes, B. S., \& Ferstl, E. C. (2013). Effects of valence and arousal on written word recognition: Time course and ERP correlates. Neuroscience Letters, 533, 90-95. doi:10.1016/j.neulet.2012. 10.054

Crites, S. L., Jr., Cacioppo, J. T., Gardner, W. L., \& Berntson, G. G. (1995). Bioelectrical echoes from evaluative categorization: II. A late positive brain potential that varies as a function of attitude registration rather than attitude report. Journal of Personality and Social Psychology, 68, 997-1013. doi:10.1037/0022-3514.68.6.997

Crowley, K. E., \& Colrain, I. M. (2004). A review of the evidence for P2 being an independent component process: Age, sleep and modality. 
Clinical Neurophysiology, 115, 732-744. doi:10.1016/j.clinph. 2003.11.021

Delaney-Busch, N. (2013). The processing of emotional features in single and primed words. Master of Science thesis, Tufts University, Medford, MA.

Delaney-Busch, N., \& Kuperberg, G. R. (2013). Friendly drug-dealers and terrifying puppies: Affective primacy can attenuate the N400 effect in emotional discourse contexts. Cognitive, Affective, \& Behavioral Neuroscience, 13, 473-490. doi:10.3758/s13415-0130159-5

Delplanque, S., Silvert, L., Hot, P., Rigoulot, S., \& Sequeira, H. (2006). Arousal and valence effects on event-related P3a and $\mathrm{P} 3 \mathrm{~b}$ during emotional categorization. International Journal of Psychophysiology, 60, 315-322. doi:10.1016/j.ijpsycho.2005. 06.006

Ding, J., Wang, L., \& Yang, Y. (2015). The dynamic influence of emotional words on sentence processing. Cognitive, Affective, \& Behavioral Neuroscience, 15, 55-68. doi:10.3758/s13415-0140315-6

Donchin, E. (1981). Presidential address, 1980. Surprise! . . Surprise? Psychophysiology, 18, 493-513. doi:10.1111/j.1469-8986.1981. tb01815.x

Donchin, E., \& Coles, M. G. H. (1988). Is the P300 component a manifestation of context updating? Behavioral and Brain Sciences, 11, 357-374. doi:10.1017/S0140525X00058027

Dunning, J. P., \& Hajcak, G. (2009). See no evil: Directing visual attention within unpleasant images modulates the electrocortical response. Psychophysiology, 46, 28-33. doi:10.1111/j.1469-8986. 2008.00723.x

Feng, C., Li, W., Tian, T., Luo, Y., Gu, R., Zhou, C., \& Luo, Y. J. (2014). Arousal modulates valence effects on both early and late stages of affective picture processing in a passive viewing task. Social Neuroscience, 9, 364-377. doi:10.1080/17470919.2014.896827

Fields, E. C., \& Kuperberg, G. R. (2012). It's all about you: An ERP study of emotion and self-relevance in discourse. NeuroImage, 62, 562574. doi:10.1016/j.neuroimage.2012.05.003

Fields, E. C., \& Kuperberg, G. R. (2015). Loving yourself more than your neighbor: ERPs reveal online effects of a self-positivity bias. Social Cognitive and Affective Neuroscience, 10, 1202-1209. doi:10.1093/ scan/nsv004

Fischler, I., \& Bradley, M. M. (2006). Event-related potential studies of language and emotion: Words, phrases, and task effects. Progress in Brain Research, 156, 185-203. doi:10.1016/S0079-6123(06) 56009-1

Fogel, A., Midgley, K., Delaney-Busch, N., \& Holcomb, P. J. (2013). Processing emotion and tabooness in a native vs. a second language: An ERP study. Poster presented at the 20th Annual Meeting of the Cognitive Neuroscience Society, San Francisco, CA.

Foti, D., \& Hajcak, G. (2008). Deconstructing reappraisal: Descriptions preceding arousing pictures modulate the subsequent neural response. Journal of Cognitive Neuroscience, 20, 977-988. doi:10. 1162/jocn.2008.20066

Foti, D., Hajcak, G., \& Dien, J. (2009). Differentiating neural responses to emotional pictures: Evidence from temporal-spatial PCA. Psychophysiology, 46, 521-530. doi:10.1111/j.1469-8986.2009. 00796.x

Gable, P. A., Adams, D. L., \& Proudfit, G. H. (2015). Transient tasks and enduring emotions: The impacts of affective content, task relevance, and picture duration on the sustained late positive potential. Cognitive, Affective, \& Behavioral Neuroscience, 15, 45-54. doi: 10.3758/s13415-014-0313-8

González-Villar, A. J., Triñanes, Y., Zurrón, M., \& Carrillo-de-la-Peña, M. T. (2014). Brain processing of task-relevant and task-irrelevant emotional words: An ERP study. Cognitive, Affective, \& Behavioral Neuroscience, 14, 939-950. doi:10.3758/s13415-013-0247-6
Greenhouse, S. W., \& Geisser, S. (1959). On methods in the analysis of profile data. Psychometrika, 24, 95-112. doi:10.1007/BF02289823

Hajcak, G., Dunning, J. P., \& Foti, D. (2009). Motivated and controlled attention to emotion: Time-course of the late positive potential. Clinical Neurophysiology, 120, 505-510. doi:10.1016/j.clinph. 2008.11.028

Hajcak, G., MacNamara, A., \& Olvet, D. M. (2010). Event-related potentials, emotion, and emotion regulation: An integrative review. Developmental Neuropsychology, 35, 129-155. doi:10.1080/ 87565640903526504

Hajcak, G., Moser, J. S., \& Simons, R. F. (2006). Attending to affect: Appraisal strategies modulate the electrocortical response to arousing pictures. Emotion, 6, 517-522. doi:10.1037/1528-3542.6.3.517

Hajcak, G., \& Olvet, D. M. (2008). The persistence of attention to emotion: Brain potentials during and after picture presentation. Emotion, 8, 250-255. doi:10.1037/1528-3542.8.2.250

Hajcak, G., Weinberg, A., MacNamara, A., \& Foti, D. (2012). ERPs and the study of emotion. In S. J. Luck \& E. S. Kappenman (Eds.), Oxford handbook of ERP components. New York: Oxford University Press.

Herbert, C., Junghöfer, M., \& Kissler, J. (2008). Event related potentials to emotional adjectives during reading. Psychophysiology, 45, 487498. doi:10.1111/j.1469-8986.2007.00638.x

Herbert, C., Kissler, J., Junghöfer, M., Peyk, P., \& Rockstroh, B. (2006). Processing of emotional adjectives: Evidence from startle EMG and ERPs. Psychophysiology, 43, 197-206. doi:10.1111/j.1469-8986. 2006.00385.x

Herring, D. R., Taylor, J. H., White, K. R., \& Crites, S. L., Jr. (2011). Electrophysiological responses to evaluative priming: The LPP is sensitive to incongruity. Emotion, 11, 794-806. doi:10.1037/ a0022804

Hinojosa, J. A., Méndez-Bértolo, C., \& Pozo, M. A. (2010). Looking at emotional words is not the same as reading emotional words: Behavioral and neural correlates. Psychophysiology, 47, 748-757. doi:10.1111/j.1469-8986.2010.00982.x

Hofmann, M. J., Kuchinke, L., Tamm, S., Võ, M. L.-H., \& Jacobs, A. M. (2009). Affective processing within 1/10th of a second: High arousal is necessary for early facilitative processing of negative but not positive words. Cognitive, Affective, \& Behavioral Neuroscience, 9, 389-397. doi:10.3758/9.4.389

Holt, D. J., Lynn, S. K., \& Kuperberg, G. R. (2009). Neurophysiological correlates of comprehending emotional meaning in context. Journal of Cognitive Neuroscience, 21, 2245-2262. doi:10.1162/jocn.2008. 21151

Ito, T. A., \& Cacioppo, J. T. (2005). Variations on a human universal: Individual differences in positivity offset and negativity bias. Cognition and Emotion, 19, 1-26. doi:10.1080/02699930441000120

Ito, T. A., Larsen, J. T., Smith, N. K., \& Cacioppo, J. T. (1998). Negative information weighs more heavily on the brain: The negativity bias in evaluative categorizations. Journal of Personality and Social Psychology, 75, 887-900. doi:10.1037/0022-3514.75.4.887

Kanske, P., \& Kotz, S. A. (2007). Concreteness in emotional words: ERP evidence from a hemifield study. Brain Research, 1148, 138-148. doi:10.1016/j.brainres.2007.02.044

Kanske, P., Plitschka, J., \& Kotz, S. A. (2011). Attentional orienting towards emotion: P2 and N400 ERP effects. Neuropsychologia, 49, 3121-3129. doi:10.1016/j.neuropsychologia.2011.07.022

Keuper, K., Zwanzger, P., Nordt, M., Eden, A., Laeger, I., Zwitserlood, P., ... Dobel, C. (2014). How "love" and "hate" differ from "sleep": Using combined electro/magnetoencephalographic data to reveal the sources of early cortical responses to emotional words. Human Brain Mapping, 35, 875-888. doi:10.1002/hbm.22220

Kissler, J., Assadollahi, R., \& Herbert, C. (2006). Emotional and semantic networks in visual word processing: Insights from ERP studies. Progress in Brain Research, 156, 147-183. doi:10.1016/S00796123(06)56008-X 
Kissler, J., \& Herbert, C. (2013). Emotion, etmnooi, or emitoon? Faster lexical access to emotional than to neutral words during reading. Biological Psychology, 92, 464-479. doi:10.1016/j.biopsycho. 2012.09.004

Kissler, J., Herbert, C., Winkler, I., \& Junghöfer, M. (2009). Emotion and attention in visual word processing - An ERP study. Biological Psychology, 80, 75-83. doi:10.1016/j.biopsycho.2008.03.004

Kissler, J., \& Koessler, S. (2011). Emotionally positive stimuli facilitate lexical decisions-an ERP study. Biological Psychology, 86, 254 264. doi:10.1016/j.biopsycho.2010.12.006

Kousta, S. T., Vinson, D. P., \& Vigliocco, G. (2009). Emotion words, regardless of polarity, have a processing advantage over neutral words. Cognition, 112, 473-481. doi:10.1016/j.cognition. 2009.06.007

Kreher, D. A., Goff, D., \& Kuperberg, G. R. (2009). Why all the confusion? Experimental task explains discrepant semantic priming effects in schizophrenia under "automatic" conditions: Evidence from event-related potentials. Schizophrenia Research, 111, 174-181. doi:10.1016/j.schres.2009.03.013

Kuppens, P., Tuerlinckx, F., Russell, J. A., \& Barrett, L. F. (2013). The relation between valence and arousal in subjective experience. Psychological Bulletin, 139, 917-940. doi:10.1037/a0030811

Lai, V. T., Hagoort, P., \& Casasanto, D. (2012). Affective primacy vs. cognitive primacy: Dissolving the debate. Frontiers in Psychology, 3, 243. doi:10.3389/fpsyg.2012.00243

Lang, P. J., Bradley, M. M., \& Cuthbert, B. N. (1997). Motivated attention: Affect, activation, and action. In P. J. Lang, R. F. Simons, \& M. T. Balaban (Eds.), Attention and orienting: Sensory and motivational processes (pp. 97-135). Mahwah: Erlbaum.

Lang, P. J., Bradley, M. M., \& Cuthbert, B. N. (2005). International Affective Picture System (IAPS): Affective ratings of pictures and instruction manual (Technical Report No. A-6). Gainesville, FL: University of Florida, Center for Research in Psychophysiology.

Lebrecht, S., Bar, M., Barrett, L. F., \& Tarr, M. J. (2012). Micro-valences: Perceiving affective valence in everyday objects. Frontiers in Psychology, 3, 107. doi:10.3389/fpsyg.2012.00107

Leite, J., Carvalho, S., Galdo-Alvarez, S., Alves, J., Sampaio, A., \& Gonçalves, Ó. F. (2012). Affective picture modulation: Valence, arousal, attention allocation, and motivational significance. International Journal of Psychophysiology, 83, 375-381. doi:10. 1016/j.ijpsycho.2011.12.005

Mather, M., \& Sutherland, M. R. (2011). Arousal-biased competition in perception and memory. Perspectives on Psychological Science, 6, 114-133. doi:10.1177/1745691611400234

Mccarthy, G., \& Donchin, E. (1981). A metric for thought-A comparison of P300 latency and reaction-time. Science, 211, 77-80. doi:10. $1126 /$ science. 7444452

Medler, D. A., \& Binder, J. R. (2005). MCWord: An on-line orthographic database of the English language (Web page). Retrieved from www. neuro.mcw.edu/mcword/

Milberg, W., \& Blumstein, S. E. (1981). Lexical decision and aphasia: Evidence for semantic processing. Brain and Language, 14, 371385. doi:10.1016/0093-934X(81)90086-9

Moreno, E. M., \& Vázquez, C. (2011). Will the glass be half full or half empty? Brain potentials and emotional expectations. Biological Psychology, 88, 131-140. doi:10.1016/j.biopsycho.2011.07.003

Nieuwenhuis, S., Aston-Jones, G., \& Cohen, J. D. (2005). Decision making, the P3, and the locus coeruleus-norepinephrine system. Psychological Bulletin, 131, 510-532. doi:10.1037/0033-2909. 131.4.510

Norris, C. J., Gollan, J., Berntson, G. G., \& Cacioppo, J. T. (2010). The current status of research on the structure of evaluative space. Biological Psychology, 84, 422-436. doi:10.1016/j.biopsycho. 2010.03.011
Okon-Singer, H., Lichtenstein-Vidne, L., \& Cohen, N. (2013). Dynamic modulation of emotional processing. Biological Psychology, 92, 480-491. doi:10.1016/j.biopsycho.2012.05.010

Olofsson, J. K., Nordin, S., Sequeira, H., \& Polich, J. (2008). Affective picture processing: An integrative review of ERP findings. Biological Psychology, 77, 247-265. doi:10.1016/j.biopsycho. 2007.11.006

Ortigue, S., Michel, C. M., Murray, M. M., Mohr, C., Carbonnel, S., \& Landis, T. (2004). Electrical neuroimaging reveals early generator modulation to emotional words. NeuroImage, 21, 1242-1251. doi: 10.1016/j.neuroimage.2003.11.007

Osgood, C. E., Suci, G. J., \& Tannenbaum, P. (1967). The measurement of meaning. Urbana: University of Illinois Press.

Osterhout, L., \& Holcomb, P. J. (1992). Event-related brain potentials elicited by syntactic anomaly. Journal of Memory and Language, 31, 785-806. doi:10.1016/0749-596X(92)90039-Z

Peeters, G., \& Czapinski, J. (1990). Positive-negative asymmetry in evaluations: The distinction between affective and informational negativity effects. European Review of Social Psychology, 1, 33-60. doi: 10.1080/14792779108401856

Polich, J. (2012). Neuropsychology of P300. In S. J. Luck \& E. S. Kappenman (Eds.), Oxford handbook of event-related potential components (pp. 159-188). New York: Oxford University Press.

Pritchard, W. S. (1981). Psychophysiology of P300. Psychological Bulletin, 89, 506-540. doi:10.1037/0033-2909.89.3.506

Recio, G., Conrad, M., Hansen, L. B., \& Jacobs, A. M. (2014). On pleasure and thrill: The interplay between arousal and valence during visual word recognition. Brain and Language, 134, 34-43. doi: 10.1016/j.bandl.2014.03.009

Redondo, J., Fraga, I., Padrón, I., \& Comesaña, M. (2007). The Spanish adaptation of ANEW (Affective Norms for English Words). Behavior Research Methods, 39, 600-605. doi:10.3758/BF03193031

Robinson, M. D., Storbeck, J., Meier, B. P., \& Kirkeby, B. S. (2004). Watch out! That could be dangerous: Valence-arousal interactions in evaluative processing. Personality and Social Psychology Bulletin, 30, 1472-1484. doi:10.1177/0146167204266647

Rousselet, G. A. (2012). Does filtering preclude us from studying ERP time-courses? Frontiers in Psychology, 3, 131. doi:10.3389/fpsyg. 2012.00131

Rozin, P., \& Royzman, E. B. (2001). Negativity bias, negativity dominance, and contagion. Personality and Social Psychology Review, 5 , 296-320. doi:10.1207/s15327957pspr0504 2

Russell, J. A. (1980). A cicumplex model of affect. Journal of Personality and Social Psychology, 39, 1161-1178. doi:10.1037/h0077714

Schacht, A., \& Sommer, W. (2009). Time course and task dependence of emotion effects in word processing. Cognitive, Affective, \& Behavioral Neuroscience, 9, 28-43. doi:10.3758/CABN.9.1.28

Schindler, S., Wegrzyn, M., Steppacher, I., \& Kissler, J. (2014). It's all in your head-How anticipating evaluation affects the processing of emotional trait adjectives. Frontiers in Psychology, 5, 1292. doi:10. 3389/fpsyg.2014.01292

Schupp, H. T., Stockburger, J., Codispoti, M., Junghöfer, M., Weike, A. I., $\&$ Hamm, A. O. (2007). Selective visual attention to emotion. Journal of Neuroscience, 27, 1082-1089. doi:10.1523/JNEUROSCI.322306.2007

Scott, G. G., O'Donnell, P. J., Leuthold, H., \& Sereno, S. C. (2009). Early emotion word processing: Evidence from event-related potentials. Biological Psychology, 80, 95-104. doi:10.1016/j.biopsycho.2008. 03.010

Taylor, S. E. (1991). Asymmetrical effects of positive and negative events: The mobilization-minimization hypothesis. Psychological Bulletin, 110, 67-85. doi:10.1037/0033-2909.110.1.67

Twomey, D. M., Murphy, P. R., Kelly, S. P., \& O'Connell, R. G. (2015). The classic P300 encodes a build-to-threshold decision variable. European Journal of Neuroscience, 42, 1636-1643. doi:10.1111/ ejn. 12936 
Võ, M. L.-H., Jacobs, A. M., \& Conrad, M. (2006). Cross-validating the Berlin Affective Word List. Behavior Research Methods, 38, 606 609. doi:10.3758/BF03193892

Vogt, J., De Houwer, J., Koster, E. H., Van Damme, S., \& Crombez, G. (2008). Allocation of spatial attention to emotional stimuli depends upon arousal and not valence. Emotion, 8, 880-885. doi:10.1037/ a0013981

Wang, L., Bastiaansen, M., Yang, Y., \& Hagoort, P. (2013). ERP evidence on the interaction between information structure and emotional salience of words. Cognitive, Affective, \& Behavioral Neuroscience, 13, 297-310. doi:10.3758/s13415-012-0146-2

Warriner, A. B., Kuperman, V., \& Brysbaert, M. (2013). Norms of valence, arousal, and dominance for 13,915 English lemmas.
Behavior Research Methods, 45, 1191-1207. doi:10.3758/ s13428-012-0314-x

Watson, D., \& Tellegen, A. (1985). Toward a consensual structure of mood. Psychological Bulletin, 98, 219-235. doi:10.1037/00332909.98.2.219

Weinberg, A., \& Hajcak, G. (2011). The late positive potential predicts subsequent interference with target processing. Journal of Cognitive Neuroscience, 23, 2994-3007. doi:10.1162/jocn.2011. 21630

Zhang, D., He, W., Wang, T., Luo, W., Zhu, X., Gu, R., . . Luo, Y. J. (2014). Three stages of emotional word processing: An ERP study with rapid serial visual presentation. Social Cognitive and Affective Neuroscience, 9, 1897-1903. doi:10.1093/scan/nst188 